\title{
Reflexiones teóricas en torno a la propuesta de recuperación del poder de compra del salario mínimo en México
}

\author{
Theoretical considerations on the proposed of recovery of purchasing \\ power of the minimum wage in Mexico
}

Carlos Guerrero de Lizardi* y Leonardo Lomelí Vanegas

Universidad Nacional Autónoma de México, México

Recibido el 3 de julio de 2015; aceptado el 26 de enero de 2016

Disponible en Internet el 12 de julio de 2016

\begin{abstract}
Resumen
Tomando como pretexto la reciente propuesta capitalina de recuperación del poder de compra del salario mínimo, se proponen algunas reflexiones en torno a las teorías de la producción y la distribución. En primer lugar se revisa el documento seminal de Cobb y Douglas (1928). A continuación se presentan algunos obstáculos en la medición de los productos marginales de los factores y un par de inconsistencias entre las teorías de la producción y distribución por un lado, y algunos ejercicios de cuantificación de las «productividades» por otro. Finalmente se retoma un modelo de competencia imperfecta que evidencia la disputa por el ingreso entre los agentes económicos. Una conclusión es que la distribución del ingreso no sigue una regla simple, sino un proceso de regateo basado en el poder de mercado, en un contexto histórico que le proporciona inercia y en el que las instituciones desempeñan un papel clave.

(C) 2016 Universidad Nacional Autónoma de México, Facultad de Contaduría y Administración. Este es un artículo Open Access bajo la licencia CC BY-NC-ND (http://creativecommons.org/licenses/by-nc-nd/4.0/).
\end{abstract}

Códigos JEL: D20; D30; D63; E64; J30

Palabras clave: Teorías de la producción y la distribución; Modelos de competencia perfecta e imperfecta; Economía política de la distribución

Abstract

* Autor para correspondencia.

Correo electrónico: cguerrero@economia.unam.mx (C. Guerrero de Lizardi).

La revisión por pares es responsabilidad de la Universidad Nacional Autónoma de México. 
Regarding the recent proposal made by the Mexico City Government about the recovery of the purchasing power of the minimum wage, some reflections on the theories of production and distribution are proposed. We first review the seminal paper written by Cobb and Douglas (1928). Some obstacles in measuring the marginal factor products, and a pair of inconsistencies between the theories of production and distribution on the one hand, and some exercises of quantifying "productivities" on the other hand, are presented. Finally, a model of imperfect competition, which displays the dispute over income between economic agents, is included. One conclusion is that income distribution does not follow a simple rule, but a bargaining process based on market power in a historical context that provides inertia and in which institutions play a key role. (C) 2016 Universidad Nacional Autónoma de México, Facultad de Contaduría y Administración. This is an open access article under the CC BY-NC-ND license (http://creativecommons.org/licenses/by-nc-nd/4.0/).

JEL classification: D20; D30; D63; E64; J30

Keywords: Production and distribution theories; Perfect and imperfect competition models; Political economy of distribution

\section{Introducción}

En el mes de agosto de 2014 el Gobierno de la Ciudad de México presentó un estudio titulado Política de recuperación del salario mínimo en México y en el Distrito Federal: Propuesta para un acuerdo nacional, elaborado por su Gabinete Económico y 9 expertos con distintas adscripciones (Antonio Azuela, Graciela Irma Bensusán, Gerardo Esquivel, Juan Carlos Moreno, Ariel Rodríguez, Enrique Provencio, Jaime Ros, Pablo Yanes y Raymundo M. Campos) (Gobierno del Distrito Federal, 2014). En un estudio que vincula el salario mínimo -y en general el resto de la estructura de remuneraciones de un país- a la llamada «productividad del trabajo» es conveniente no perder de vista la distinción entre las teorías de la producción y las teorías de la distribución que le subyacen. Nuestra intención aquí es hacerlo.

La presente investigación está estructurada en 5 secciones. En la primera se realiza una mínima exposición de la propuesta capitalina. En la segunda sección se comenta, desde una perspectiva teórico-histórica, el documento seminal escrito por Cobb y Douglas (1928). Sin abandonar la perspectiva neoclásica, en la tercera se presentan algunos obstáculos prácticos en la medición de los productos marginales de los factores de la producción y un par de inconsistencias entre las teorías de la producción y distribución por un lado, y algunos ejercicios de cuantificación de las «productividades» por otro. En la cuarta sección se retoma un modelo de competencia imperfecta poskeynesiano que determina la cantidad de bienes y servicios disponibles a partir de la cantidad de empleo y la productividad aparente del trabajo, y pone en evidencia la disputa por el ingreso entre los agentes económicos. El documento cierra con algunas reflexiones finales.

\section{Mínima revisión de la propuesta capitalina}

Con base en un esfuerzo serio de recopilación de estadísticas oficiales mexicanas y de otros países, el documento presentado por el Gobierno capitalino propuso, en primer lugar, un conjunto de hechos estilizados sobre la trayectoria de los salarios mínimos y las «productividades del trabajo» no solo para México sino para un numeroso conjunto de países. En este sentido, adoptó atinadamente una perspectiva histórica e internacional para revisar las variables clave de su estudio.

Las siguientes 2 gráficas (figs. 1 y 2) revelan el comportamiento del salario mínimo en México y su insuficiencia para acceder a las canastas definidas por el Consejo Nacional de Evaluación de 


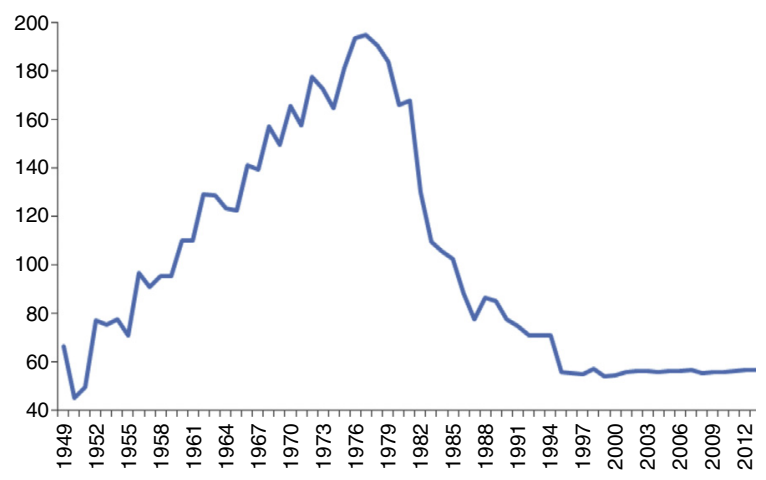

Figura 1. Salario mínimo real nacional 1949-2014 (pesos por día, base 2010=100).

Fuente: Gobierno del Distrito Federal (2014, p. 10).

la Política de Desarrollo Social -organismo público descentralizado de la Administración Pública Federal-, según la metodología oficial para la medición de la pobreza multidimensional.

Es de conocimiento común la pérdida de poder de compra del salario mínimo real de alrededor del $75 \%$ a lo largo de las últimas 3 décadas. Puesto con otra perspectiva, el salario pagado actualmente tiene el mismo nivel que el correspondiente a los últimos años 40. Creemos que nadie se atrevería a afirmar que la productividad del trabajo en general, y la observada en la producción de bienes salario en particular, se ha estancado a lo largo del periodo que abarca la gráfica anterior, esto es, en 66 años. Debe resulta evidente entonces que el meollo de la fijación del salario mínimo no radica en la esfera de la producción sino en la correspondiente a la distribución.

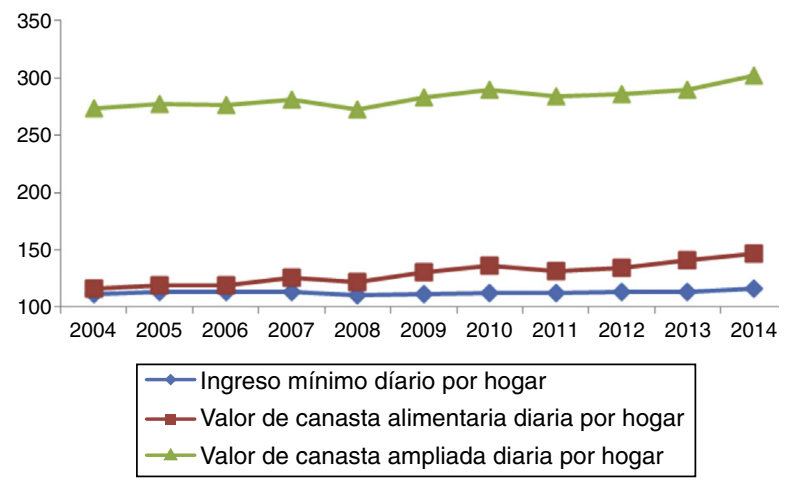

Figura 2. Evolución del costo de las canastas alimentaria diaria y ampliada, e ingreso mínimo diario por hogar2004-2014 (pesos por día, base $2010=100$ ).

Fuente: Gobierno del Distrito Federal (2014, p. 14).

En el documento del Gobierno de la Ciudad aparece la siguiente nota a propósito de la gráfica: «deflactado con el IPC $2010=100$. Para observar la evolución de la canasta básica se tomó como base la canasta alimentaria y la canasta ampliada que utiliza CONEVAL para establecer líneas de bienestar y de pobreza. La canasta ampliada representa la canasta alimentaria y la canasta no alimentaria básica. Se consideró un hogar promedio como una familia de cuatro integrantes: dos que aportan un ingreso mínimo y dos dependientes económicos. El ingreso mínimo corresponde a dos salarios mínimos». 


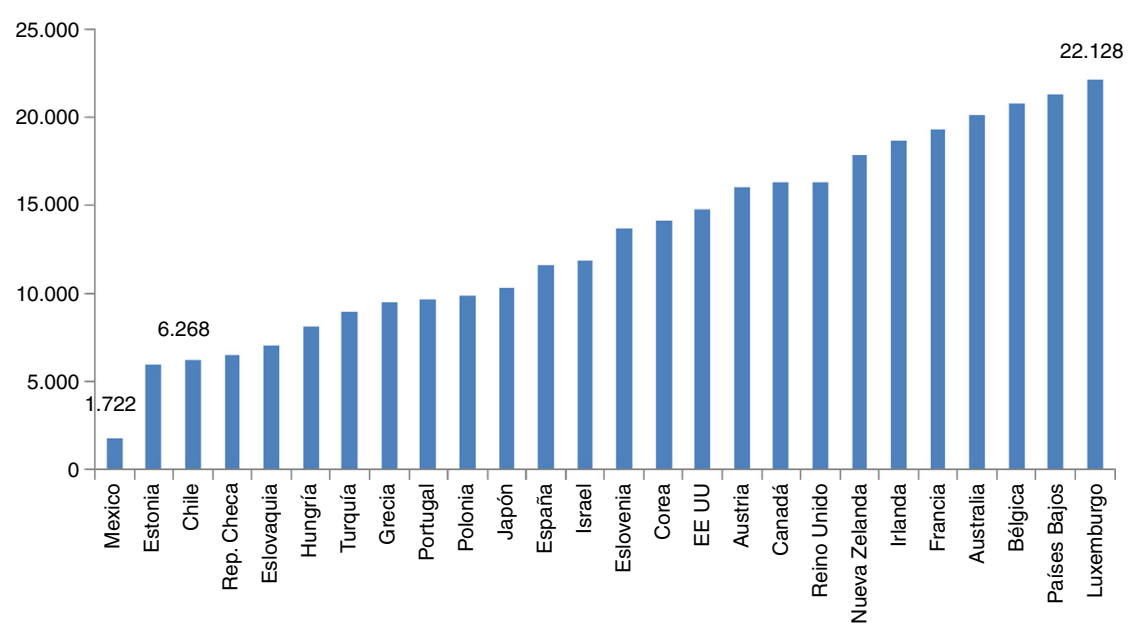

Figura 3. Salario mínimo real anual 2013 (en dólares PPC).

Fuente: elaboración propia con datos de la OCDE (OECD, 2012).

La información contenida en las figuras 1 y 2 basta y sobra para que el Gobierno de la Ciudad de México plantee lo siguiente. El primer paso en la recuperación del poder de compra del salario mínimo representa incrementarlo 15.57 pesos al día, o lo que es lo mismo, aumentarlo de 67.29 pesos a 82.86 pesos al día entre el 2014 y el 2015. Un incremento equivalente al precio de un litro de leche al día -suponiendo una típica estructura familiar de 4 integrantes, 2 de los cuales trabajan y obtienen un salario mínimo-, permitiría que esa familia estándar alcanzara un ingreso mensual acumulado igual al valor de la canasta alimentaria mensual fijada por el CONEVAL. Con otras palabras, se trata de establecer un salario mínimo que garantice que una familia -con 2 miembros en el sector formal de la economía-, rebase la definición más elemental de pobreza -llamada pobreza extrema por el propio Consejo-; o si se prefiere, llanamente cumplir con el artículo 123 de la Constitución que prescribe «los salarios mínimos generales deberán ser suficientes para satisfacer las necesidades normales de un jefe de familia, en el orden material, social y cultural, y para proveer a la educación obligatoria de los hijos.» Nótese que el citado artículo se refiere a un salario mínimo por familia.

Ahora bien, la política de fijación del salario mínimo real representa un componente relevante del paquete de políticas económicas instrumentadas en un país -se haga explícito o se niegue. Es nuestra hipótesis que, en México, el acelerado crecimiento económico con disminución de la pobreza, y con baja y controlada inflación durante las primeras décadas de la posguerra tuvo como un componente energético el jugoso crecimiento del poder de compra de los trabajadores. En contraste, a raíz del desconcierto económico observado a mediados de los setenta y desde los ochenta, la política económica nacional ha descansado en la premisa de que los salarios bajos constituyen tanto el ancla de la inflación como el instrumento clave de competitividad espuria de nuestro país (Guerrero, 2011). Así entendemos la reticencia del grueso de las autoridades económicas y financieras a siquiera discutir inicialmente la propuesta capitalina.

Las siguientes 2 gráficas justifican que en el estudio en revisión se hable de México como un caso no solo «atípico» sino «excéntrico de inercia» a escala internacional (figs. 3 y 4 ).

Por lo menos entre el 2002 y el 2011, y dada la canasta de países incluida en la anterior gráfica, en México no solo se pagó uno de los salarios mínimos más bajos de la región, sino que se presentó 


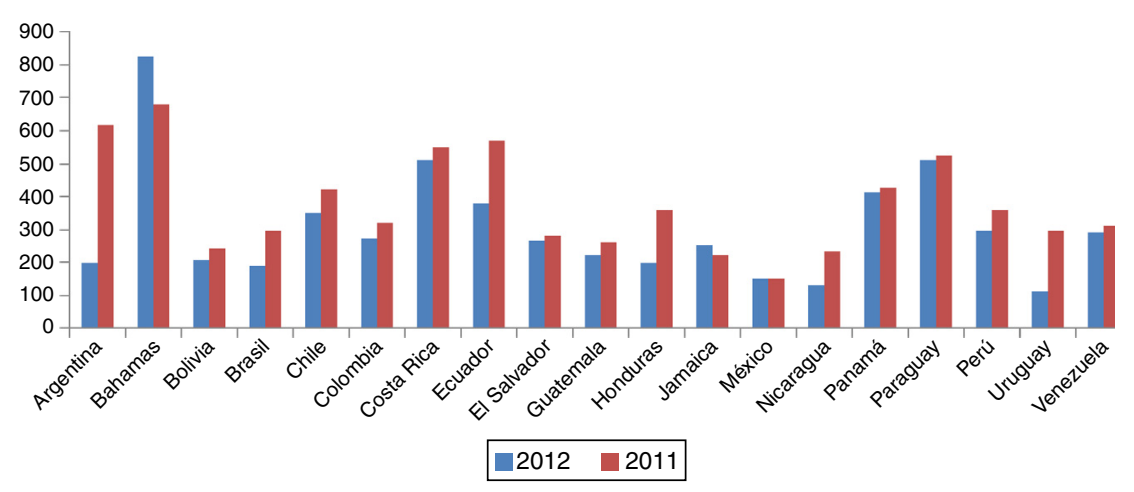

Figura 4. Salario mínimo real mensual 2002 y 2011 (dólares PPC).

Fuente: elaboración propia con datos de la CEPAL.

como el único país en el que se congeló. Solo para hacer acopio de evidencia empírica adicional, las siguientes 2 gráficas muestran que no existe ningún argumento supuestamente «técnico» para justificar la caída del salario mínimo real, y mucho menos la oposición a una política de recuperación de su poder de compra (figs. 5 y 6).

La propuesta de recuperación del salario mínimo contenida en el estudio en juego incluyó argumentos teóricos variopintos, tanto microeconómicos como macroeconómicos, incluyó también algunos ejercicios estadísticos, y tanto o más importante, empleó una perspectiva histórica e internacional. Para cerrar el presente apartado baste señalar que, en un reciente Informe mundial sobre salarios de la Organización Internacional del Trabajo, se afirma que «en México el salario mínimo está por debajo de los niveles de mercado, aún para los trabajadores no calificados» (Gobierno del Distrito Federal, 2014, p. 20).

\section{Breve nota sobre Cobb y Douglas (1928)}

En el encuentro de la Sociedad de Economistas Americanos de 1927, Paul H. Douglas presentó un documento titulado «A theory of production», escrito con Charles Cobb. Se trata de un documento seminal porque utilizó los mínimos cuadrados ordinarios para estimar una función

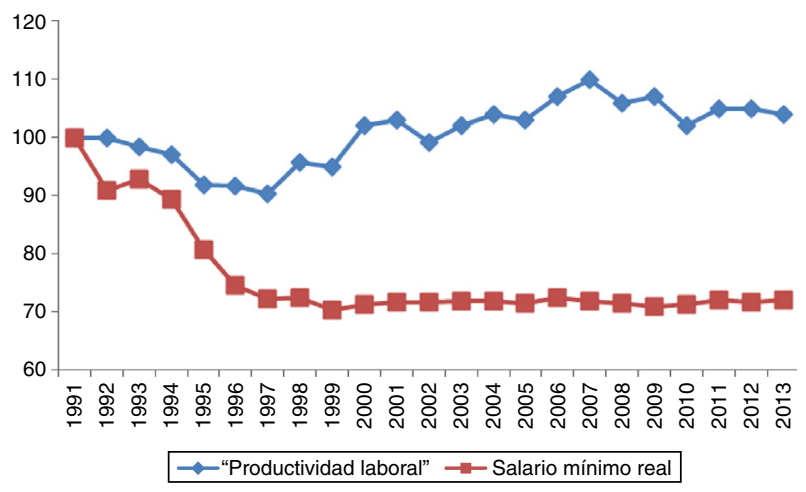

Figura 5. Índice del salario mínimo real y de la «productividad laboral» 1991-2013 (1991=100). Fuente: elaboración propia con datos de la OCDE. (OECD, 2012). 


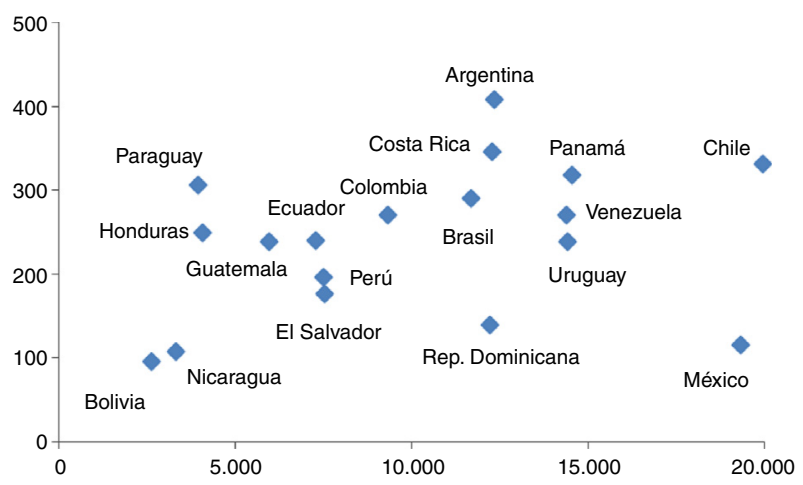

Figura 6. «Productividad laboral» (dólares de 2005, eje horizontal) y salarios mínimos mensuales (dólares corrientes, eje vertical) en América Latina 2010.

Fuente: elaboración propia con datos de la OCDE (OECD, 2012).

de producción por primera ocasión en la literatura. Biddle (2012, pp. 225-6) nos explica qué se entendía por las teorías de la producción y de la distribución en aquellos tiempos, y tanto o más revelador, la falta de consenso entre la comunidad de economistas respecto a los determinantes de la distribución:

«In the 1920s, a "theory of production" was understood to be an explanation of the determinants of the level of output. The amount produced, it was generally agreed, depended upon the level of technological knowledge and the quantities of the factors of production employed... A theory of distribution, on the other hand, explained the determination of the division of output between various members of society. . . By the 1920s, the economics profession displayed no consensus regarding the appropriate approach to theorizing about distribution, with approaches based on the marginal productivity principle competing with those rooted in the classical tradition and those emphasizing the ways in which social and economic institutions influenced the bargaining power of various groups.»

$\mathrm{Al}$ amable lector no le debe caber duda respecto al envidiable ambiente académico de principios del siglo pasado, en el que perfectamente se diferenciaba la producción -o puesto coloquialmente el tamaño del pastel- y la distribución; y también se distinguían las hipótesis en competencia o rivales en torno al tamaño de las «rebanadas del pastel». Así por ejemplo Sumner Slichter -el comentarista asignado para discutir el documento presentado por Douglas-, tuvo una participación decididamente crítica hacia el documento seminal. Biddle (2012 pp. 225-6) escribió al respecto:

«The bulk of his remarks were devoted to detailing problems with the index of fixed capital constructed by Douglas, but his complaints went beyond issues of data quality as he believed the entire project to be wrong-headed. Despite the fact that the marginal productivity theory was not explicitly mentioned in the paper, Slichter thought he could see a hidden agenda, and he did not approve: 'Professors Cobb and Douglas conclude that it has been statistically demonstrated that the relationship between the agents of production on one hand and the volume of output on the other meets the requirements of the marginal productivity hypothesis.' Slichter disputed this specific claim and argued more generally that marginal productivity theory had little to offer as a framework for thinking about distribution.» 
De la siguiente cita del último documento publicado por Douglas (1975, p. 914) se infiere que no fue sino hasta la década de los años cincuenta cuando el marginalismo desplazó al resto de las teorías de la distribución, por lo menos en el grueso de la comunidad de economistas americanos:

«A considerable body of independent work tends to corroborate the original Cobb-Douglas formula, but, more important, the approximate coincidence of the estimated coefficients with the actual shares received also strengthens the competitive theory of distribution and disproves the Marxian. Many of the original objections have been answered. Some remain. . . I, therefore, still appeal to the younger generation of economists and statisticians for help. A quarter of a century ago, there would have been distinct professional risks in such a venture. The opponents of the production function were eminent, powerful, and determined. No such danger exists today. The times favor such studies. I hope they may be made.»

Así fue como, al final de su carrera, el citado autor publicó un documento en el que conscientemente empalmó las teorías de la producción y distribución. En ese mismo año Paul H. Douglas falleció. No es posible entender por qué lo hizo, pero queremos señalar que vale la pena leer su obra y sus memorias. Como joven doctorante trabajó como negociador entre trabajadores y dueños de empresas, y votó por el ya desaparecido Partido Socialista Americano -el que, a propósito, se disolvió a principios de los años cincuenta por riñas entre corrientes. Así nos explicamos su claridad inicial para distinguir entre las teorías de la producción y la distribución, pero también su posterior fobia hacia las ideas marxistas blandidas por el llamado socialismo soviético y su bloque geopolítico. Baste como hipótesis señalar, si se nos permite la expresión, que la comunidad de economistas se «engolosinó» con la herramienta estadística -los mínimos cuadrados ordinarios-, y sus aparentes buenos resultados. El profesor Mark Lavoie (2008 pp. 2-3) lo resume de manera divertidamente demoledora:

«The word artefact carries several definitions. The most common definition, relevant to science, says that an artefact, or artifact, is a spurious finding caused by faulty procedures. It is a finding that does not really exist but that was created inadvertently by the researcher. In particular, we shall see that neoclassical economists claim to measure output elasticities with respect to capital and labour, whereas in reality they are estimating the profit and wage shares in income. The word artifact is also used in the fantasy literature. In the fantasy and sorcery literature, an artifact is a magical tool with great power, like a magic wand. This definition seems to be just as relevant to the neoclassical production function. Correlation coefficients obtained with regressions of Cobb-Douglas production functions miraculously approach unity, and all the predictions that can be drawn from a model of perfect competition applied to the Cobb-Douglas production function are usually verified, even when we know that these conditions do not hold. In other words, the neoclassical production functions and their derived labour demand functions are not behavioral concepts that can be empirically refuted. Their magical power is enormous!»

\section{Marginalismo: tras la búsqueda de una regla simple}

El punto de partida es una función de producción microeconómica:

$$
Y_{i}=f\left(K_{i}, L_{i}\right)
$$

En donde del lado izquierdo aparece el nivel de producto, y del lado derecho el acervo de capital y el trabajo, y las 3 variables aparecen en términos físicos. Utilizamos el subíndice «i» para dejar 
claro que la función de producción no se refiere a una empresa sino que refleja las condiciones técnicas de una de sus líneas de producción. Un supuesto implícito es que la organización de la producción garantiza el uso eficiente tanto del capital como del trabajo. Adicionalmente, nosotros suponemos un escenario sin cambio técnico solo como mecanismo de simplificación. A partir de la ecuación (1) es inmediato calcular los productos marginales del capital y del trabajo de manera independiente. Parece entonces que, «sin mayor preámbulo», se puede transitar de la producción a la distribución a la neoclásica. Pero no es el caso. Revisemos el asunto con cuidado.

En primer lugar, en sus términos más llanos la función de producción es una perogrullada que dice «para producir se requieren factores de la producción». Puesto con otras palabras, una función de producción refleja simplemente una identidad contable entre el valor agregado por un lado, y las cantidades de capital y trabajo multiplicados por sus ingresos respectivamente, por otro. Respecto a este punto existe consenso entre los economistas. Hasta aquí la teoría de la producción neoclásica.

En segundo lugar, solo suponiendo un escenario de competencia perfecta -que incluye a todos y cada uno de los mercados de bienes y servicios, destacadamente los correspondientes a los factores de la producción-, los salarios y las ganancias se fijan a partir de los productos marginales del trabajo y el capital. En este sentido, en la función de producción Cobb-Douglas no solamente encontramos estética matemática sino una genuina aspiración compartida por las ciencias sociales $-\mathrm{y}$ suponemos que por el resto de las ciencias-, a saber, la construcción de una regla simple para explicar, en este caso, la distribución del ingreso. A propósito, bajo competencia perfecta otro resultado tanto o más atractivo que no debemos olvidar es que la economía ocupa plenamente sus recursos productivos.

Puesto formalmente, el producto marginal del trabajo se define como:

$$
\frac{\Delta Y_{i}}{\Delta L_{i}}
$$

El incremento del producto derivado de una hora de trabajo adicional o la contratación de un trabajador adicional está puesto en términos de unidades físicas de «i», por lo que para obtener el salario nominal hace falta multiplicar a (2) por el precio del bien o servicio producido:

$$
w_{n o m, i}=\left(\frac{\Delta Y_{i}}{\Delta L_{i}}\right) \times P_{i}
$$

$\mathrm{Si} \ll P_{i} \gg$ es un precio fijado bajo competencia perfecta, entonces en el modelo teórico se obtiene que el salario nominal y el producto marginal del trabajo son magnitudes iguales ${ }^{1}$. Si no es el caso, entonces simple y llanamente se rompe la regla marginalista. Y por adelantado no se sabe en qué dirección y en qué medida. Bajo un escenario de competencia imperfecta y siendo optimistas la ecuación (2) se reduce a una «regla de dedo gordo»-si se nos permite el anglicismo.

A estas alturas aceptando sin conceder la hipótesis marginalista, en tercer lugar debe quedar claro que el salario real determinado con base en el precio del bien o servicio producido y el

\footnotetext{
${ }^{1}$ En el caso del Sistema de Cuentas Nacionales estructura 2003, el INEGI (2011, sección primera titulada Presentación, sin número de página, y p. 31) nos informa que en la economía mexicana se producían, en términos de diversidad, veinticinco mil bienes y servicios distintos, y se importaron, por fracción arancelaria y a nivel de pedimento, doce mil productos. Simplificando al extremo el análisis, por ej. dejando de lado, si el gentil lector puede, las dimensiones espacial y temporal, podemos decir que hablamos entonces de treinta y siete mil mercados en los que se supone competencia perfecta. Ni más ni menos.
} 


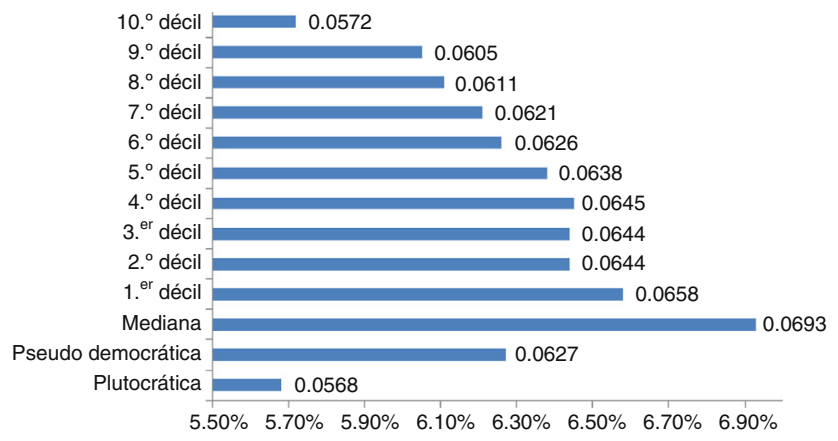

Figura 7. Tasas de crecimiento de los precios de la canasta básica utilizando distintos ponderadores según las Encuestas Nacionales de Ingreso y Gasto de los Hogares 2002-2009.

Fuente: Guerrero (2010).

salario real determinado con base al índice de precios al consumidor (IPC), son evidentemente magnitudes distintas:

$$
\frac{w_{\text {nom }, i}}{P_{i}} \neq \frac{w_{\text {nom }, i}}{I P C}
$$

Con otras palabras, la ecuación anterior deja claro que la determinación del incremento a los salarios nominales utilizando como referencia la tasa de crecimiento del IPC -operación bastante común en las negociaciones entre los factores de la producción- es una flagrante violación de la «regla» de dedo gordo.

En cuarto lugar, el IPC es un índice de Laspeyres en el que los ponderadores de los bienes y servicios consumidos se calculan utilizando las medias de gasto, esto es, sumando el gasto en cada uno de los mismos y dividiendo entre el gasto total de las familias; a propósito, este método de agregación se conoce en la literatura como método «plutocrático», en oposición a otros métodos, por ej. «democrático» (Guerrero, 2014). Mary Kokoski (2003, p. 4), investigadora de la Agencia de Estadísticas Laborales de nuestro vecino país del norte, lo resume así: «on one hand the plutocratic aggregation means 'one dollar, one vote', so it treats all dollars of expenditure equally, and on the other the democratic aggregation states 'one household, one vote'.»

En la literatura existen propuestas para utilizar otros métodos de agregación. Prais (1959) propuso, como se acaba de comentar, un enfoque democrático; el Nobel Arrow (1958) recomendó realizar tantas mediciones de la inflación como número de deciles; y más recientemente Pollak (1998) sugirió utilizar la mediana de la distribución del gasto. El meollo es que utilizar el IPC como referencia para determinar los incrementos salariales representa suponer que el patrón de consumo del grueso de las familias, en nuestro caso mexicanas, es similar al observado en el décimo decil; la siguiente gráfica lo ilustra (fig. 7):

En quinto lugar, en la función de producción la causalidad es unidireccional e inequívoca, de los «inputs» al «output» evidentemente, y los productos marginales constituyen coeficientes técnicos, esto es, valores constantes en el corto plazo. Sin embargo, en estudios empíricos sobre las así llamadas «productividades» se destacan sus comportamientos procíclicos, lo que representa un sinsentido desde el modelo teórico canónico. Solo como botones de muestra señalemos que en el estudio capitalino se habla de las «fluctuaciones de la productividad por hora», y que en un reciente estudio de la OCDE (OECD, 2012) se habla de los «patrones cíclicos de la productividad del trabajo y multifactorial». Se reconoce por tanto que las economías reales no son escenarios de 
competencia perfecta, por lo que ni funcionan bajo pleno empleo ni los factores de la producción obtienen sus productos marginales. Si se nos permite la expresión se adopta así un keynesianismo «de closet».

En la misma dirección recordemos que la introducción de los así llamados «salarios de eficiencia» representa el rechazo tácito del principio marginalista en el sentido de que se afirma que los trabajadores mejor pagados serán los más productivos. Colateralmente se reconoce así el incumplimiento del supuesto implícito de eficiencia en la ecuación (1).

Finalmente, estamos seguros que el atento lector observó que repetidamente entrecomillamos «productividad del trabajo»-a pesar de su uso común y corriente. El meollo es que desde la función de producción lo que rigurosamente se obtiene -dividiendo simplemente a (1) entre el factor trabajo- es la productividad aparente del trabajo. Reescribir así a la función de producción nos revela que la productividad aparente del trabajo depende de la relación capital-trabajo. Otra vez, la así mal llamada productividad del trabajo -puesta por los economistas de libro de texto como condición del incremento del salario mínimo-, se revela no como dependiente del esfuerzo individual -recordemos una vez más que un supuesto del modelo es que la organización de la producción garantiza la eficiencia en el uso de los factores de la producción-, sino dependiente de la dotación de capital por unidad de trabajo.

\section{Un modelo de competencia imperfecta poskeynesiano}

A continuación se presenta un escenario teórico alternativo que, creemos, sirve para entender 2 hechos fundamentales de cualquier economía de mercado (Guerrero, 2009). El primero, basado en una identidad contable hace notar que la cantidad de bienes y servicios disponible en una economía depende de la cantidad de trabajo y su productividad aparente. El segundo, la distribución de esa cantidad de bienes y servicios no sigue una regla simple, sino que es el resultado de un proceso de regateo entre los agentes económicos en el que su poder de mercado desempeña un papel clave, en un contexto histórico que le proporciona mucha inercia y en el que las instituciones representan el telón de fondo, para bien y para mal.

El siguiente conjunto de ecuaciones corresponde a un modelo de competencia imperfecta a la poskeynesiana, esto es, a una economía en la que algunas empresas, digamos las dominantes en cada línea de producción, son «hacedoras de precios», en el sentido que fijan su propio margen de ganancia, y el resto más bien «sigue los precios». El atento lector descubrirá que una buena parte de este modelo está basado en identidades contables.

Iniciamos con una economía cerrada. El costo laboral unitario equivale al costo salarial por unidad de producto:

$$
\frac{W E}{Y}=\frac{W}{L P}
$$

Donde la productividad aparente del trabajo (LP) se define como:

$$
L P=\frac{Y}{E}
$$

Siguiendo la regla del margen de ganancia $(\mu)$, el precio se determina así:

$$
P=(1+\mu)\left(\frac{W}{L P}\right)
$$




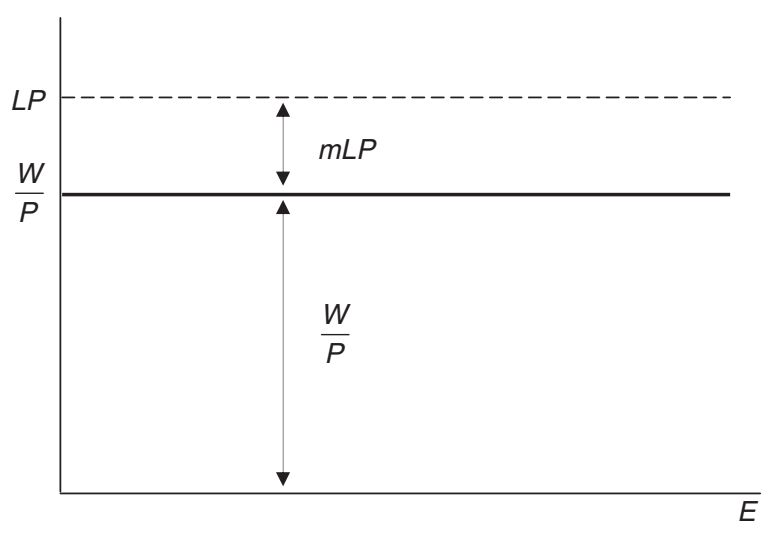

Figura 8. Determinación del salario real en una economía cerrada.

Dicho brevemente, $\mu$ depende del poder de mercado de las empresas líderes, y se supone relativamente estable. Otra manera de expresar a (7) es:

$$
P=\left(\frac{1}{1-m}\right)\left(\frac{W}{L P}\right)
$$

En donde:

$$
m=\frac{\mu}{(1+\mu)}
$$

Reescribiendo a (8):

$$
P=m P+\frac{W}{L P}
$$

Esto es, el precio se descompone en 2 partes. La primera corresponde al margen de ganancia unitario, y la segunda al costo laboral unitario que, como vimos, depende inversamente de la productividad aparente del trabajo.

Si dividimos entre el precio y multiplicamos por la productividad aparente del trabajo llegamos a:

$$
L P=m L P+\frac{W}{P}
$$

La ecuación anterior pone de relieve la disputa por el producto generado por trabajador, o puesto con otras palabras, su igualdad con la suma del beneficio real obtenido por trabajador contratado y el salario real por trabajador, lo que representa el límite fijado por la productividad aparente del trabajo a las aspiraciones económicas de los participantes en juego. Dadas las variables contenidas en la ecuación anterior, a saber, el margen de ganancia, la productividad aparente del trabajo y el salario nominal, la fijación del precio implica un determinado nivel de salario real:

$$
w=\frac{W}{P}=L P(1-m)
$$

Con otras palabras, la ecuación anterior muestra que lo que se «embolsan» unos lo dejan de «recibir» otros, en oposición al planteamiento marginalista. Puesto gráficamente (fig. 8): 
En una economía abierta tenemos que reescribir a (10) de la siguiente manera:

$$
P=m P+\frac{W}{L P}+\frac{P * e}{M P}
$$

El tercer término representa al costo unitario de los insumos importados en moneda local, es decir, $\left(\frac{1}{M P}\right)$ representa el costo, $M P$ su «productividad», y $(P * e)$ la traducción del precio externo utilizando el tipo de cambio nominal. Dividiendo por el precio y utilizando la definición más común del tipo de cambio real $\left(\theta=\frac{P * e}{P}\right)$, esto es, un incremento de teta implica dar más unidades monetarias locales por un dólar, o lo que es equivalente, ganar competitividad, y lo contrario, podemos reescribir a (13) de sugerentes maneras:

$$
\begin{aligned}
& 1=m+\frac{w}{L P}+\frac{\theta}{M P} \\
& L P=m L P+w+\theta\left(\frac{L P}{M P}\right) \\
& w=L P-m L P-\theta\left(\frac{L P}{M P}\right)
\end{aligned}
$$

La ecuación (15) revela que, en la disputa por el producto por trabajador en una economía abierta, surge un tercer participante, aproximado por el costo unitario real de los insumos importados, y que la satisfacción de las exigencias de los participantes tiene como variable clave, o límite objetivo, la productividad aparente del trabajo. Por su parte, la expresión (16) coloca al salario real como una variable residual. Creemos que este modelo sirve para explicar el conjunto de hechos estilizados contenidos en la primera sección del presente documento, esto es, para entender la evolución conjunta del salario mínimo por un lado, y de la productividad del trabajo, de los márgenes de ganancia, y la competitividad espuria de la economía mexicana por el otro.

\section{Reflexiones finales}

Como telón de fondo tenemos que recordar que el reducido crecimiento económico de las últimas décadas es, en buena medida, resultado de la pérdida de poder adquisitivo de los trabajadores asalariados. La economía mexicana ha sustituido el estímulo de la demanda interna por la demanda externa originando una dependencia, fragilidad y pérdida de arrastre nunca antes vistas, o puesto con otras palabras, una trayectoria económica que se tiene que romper si queremos crecer más y mejor. Y los niveles de pobreza e informalidad son expresiones de la misma política salarial, es decir, son fenómenos causados por el propio modelo económico implementado desde hace décadas. Según el CONEVAL (2013, p. 15): «entre 2010 y 2012, la proporción de personas en pobreza en el país pasó del 46.1 al $45.5 \%$. Si bien el cambio demostró no ser estadísticamente significativo, esto implicó que, como resultado del crecimiento poblacional, la estimación puntual del número de personas en pobreza pasara de 52.8 a 53.3 millones de personas.» $\mathrm{Y}$ en el 2012 según el INEGI, 19.6 millones de personas ocupadas se encontraban en la informalidad, y 29.3 millones en la economía formal. A propósito, en su definición de informalidad el INEGI no incluye a los informales que trabajan en el sector formal, por lo que cabe suponer una subestimación de su número. Desde nuestro punto de vista está claro que es necesario superar ya los traumas pasados, resulta imprescindible instrumentar nuevas políticas económicas acompañadas 
de nuevos diseños institucionales, para enfrentar los enormes problemas nacionales de cara a la globalización que opera como realidad mundial.

Recomendamos en todo momento tener en mente la distinción entre producción y distribución. En el caso de la función Cobb-Douglas lo anterior se traduce en no perder de vista los supuestos que permiten establecer su regla simple de distribución, entre otras características de su construcción. Curiosamente en otros pensamientos ocurre algo similar. Como los neoclásicos típicamente empalman sus teorías de la producción y la distribución, los economistas poskeynesianos critican la función de producción -que no expresa otra cosa más que una identidad contable- como medio para atacar la propuesta marginalista. Y en los economistas marxistas pasa algo similar, suponen que si logran construir «incontrovertible» evidencia empírica respecto a la vinculación de los valores y los precios, entonces se daría por buena la teoría del plusvalor marxista, léase su teoría de la explotación del trabajo vivo. ¡Para todas y cada una de las escuelas de pensamiento citadas, no es el caso, esto es, la validez teórica de una hipótesis por ej. relativa a la esfera de la producción, no se traslada automáticamente a otra hipótesis, por ej. sobre la distribución! Si se prefiere, diríamos que lo anterior merece por lo menos un debate profundo en torno a la metodología de la ciencia, en economía en particular, y en otras ciencias sociales en general-ciencias no experimentales, esto es, que requieren la construcción de simplificaciones imperfectas de la realidad llamadas modelos.

El documento presentado por el Gobierno capitalino es excepcional en muchos sentidos. Se trata de una iniciativa desde el poder político para construir conjuntamente una política económica, en este caso de recuperación del poder de compra del salario mínimo de los trabajadores con el menor poder de negociación del mercado. El tema entrará y saldrá estacionalmente de la discusión pública -previo a las elecciones intermedias de 2015 ya abrió la discusión pública en nuestro país, y estamos seguros que constituirá el tema de política pública antes de las elecciones presidenciales de 2018-, y dependerá de la actuación de muchos y variados actores económicos y políticos llegar a buen puerto.

\section{Referencias}

Arrow, K. J. (1958). The measurement of price changes. The relationship of prices to economic stability and growth. Joint Economic Committee U. S. Congress, U. S. Government Printing Office, pp. 77-87.

Biddle, J. (2012). The introduction of the Cobb-Douglas regression. Journal of Economic Perspectives, 26(2), 223-236. http://dx.doi.org/10.1257/jep.26.2.223

Cobb Ch, W. y Douglas, P. H. (1928). A theory of production. American Economic Review, Supplement, Papers and Proceedings of the Fortieth Annual Meeting of the American Economic Association, 18(1), 139-165.

CONEVAL (2013). Informe de la pobreza en México 2012. México: CONEVAL.

Douglas, P. H. (1975). The Cobb-Douglas production function once again: Its history, its testing, and some new empirical values. Journal of Political Economy, 84(5), 903-916. http://dx.doi.org/10.1086/260489

Gobierno del Distrito Federal (2014). Política de recuperación del salario mínimo en México y en el Distrito Federal: Propuesta para un acuerdo nacional, Ciudad de México.

Guerrero, C. (2009). Determinantes económicos del salario mínimo en países pequeños y abiertos: una aplicación para Centroamérica. Estudios y Perspectivas, (118).

Guerrero, C. (2010). Alternative consumer price indexes for Mexico. Center for International Development Working Paper, n. ${ }^{\circ}$ 42, John F. Kennedy School of Government, Harvard University.

Guerrero, C. (2011). Determinantes económicos de las remuneraciones en las manufacturas mexicanas. Panorama Económico, 6(12), 41-56.

Guerrero, C. (2014). A case of 'No man's land' in economics: The theory of price indexes and its applications. International Journal of Pluralism and Economics Education, 5(2), 144-156.

Kokoski, M. (2003). Alternative consumer price index aggregations: Plutocratic and democratic approaches. BLS Working Paper, (n. $\left.{ }^{\circ} 370\right)$. 
Lavoie, M. (2008). Neoclassical empirical evidence on employment and production laws as artefact. Robinson WP $n .^{\circ}$ 08-09, apareció también en Economía Informa, n.o 351, pp. 9-36.

OECD (2012). OECD compendium of productivity indicators 2012. OECD Publishing.

Pollak, R. A. (1998). The consumer price index: A research agenda and three proposals. Journal of Economic Perspectives, 12(1), 69-78. http://dx.doi.org/10.1257/jep.12.1.69

Prais, S. J. (1959). Whose cost of living? The Review of Economic Studies, 26(2), 126-134. http://dx.doi.org/ $10.2307 / 2296170$ 